\title{
Case Report: Uterine Adenocarcinoma Metastasis to the Skull Base and Cervical Spine Presenting with Pathological Fracture and Myelopathy
}

\author{
Paul E. Kaloostian ${ }^{1}$, Han Chen $^{2 *}$, Martina Stippler ${ }^{2}$ \\ ${ }^{1}$ The Johns Hopkins University Department of Neurosurgery, Baltimore, USA \\ ${ }^{2}$ University of New Mexico Department of Neurosurgery, Albuquerque, USA \\ Email: "pkaloos1@jhmi.edu
}

Received February 20, 2013; revised March 20, 2013; accepted April 4, 2013

Copyright (C) 2013 Paul E. Kaloostian et al. This is an open access article distributed under the Creative Commons Attribution License, which permits unrestricted use, distribution, and reproduction in any medium, provided the original work is properly cited.

\begin{abstract}
Objective: The authors report a rare case of uterine adenocarcinoma metastasis to both the skull base and cervical spine presenting with pathological fracture and myelopathy. Methods: We report the case of a 43-year-old Asian female with widely metastatic uterine adenocarcinoma who over the course of 3 years was diagnosed with a large clival mass and more recently a cervical spine pathological fracture presenting with myelopathy. Results: This patient underwent multiple procedures over a three-year period, including total hysterectomy with tumor debulking, endonasal biopsy of clival mass and most recently cervical corpectomy and fusion. She has received chemotherapy and radiation therapy for treatment of the unresectable clival mass and has done quite well despite having this pathology. Conclusion: This is the first documented case of clival involvement of endometrial adenocarcinoma. Additionally, this is a unique case of spinal metastatic endometrial adenocarcinoma presenting with pathological fracture and myelopathy.
\end{abstract}

Keywords: Cancer; Metastasis; Pathological Fracture; Myelopathy; Skull Base

\section{Introduction}

Endometrial cancer is the most common gynecologic malignancy in the United States. An estimated 40,100 cases are diagnosed annually, leading to 7470 deaths. It is the fourth most common cancer, accounting for $6 \%$ of female cancers, following breast, lung and colorectal cancer [1]. Endometrial adenocarcinoma is the most common cancer in the female reproductive system. We report a rare case of uterine adenocarcinoma metastasis to both the skull base and cervical spine presenting with pathological fracture and myelopathy.

\section{Case Reports}

\section{Case Presentation}

We report the case of a 43-year-old Asian female who presented two years ago with vaginal bleeding and chronic pelvic pain. She was diagnosed with stage 4A locally advanced endometrial adenocarcinoma with mucinous differentiation and underwent radical hysterectomy and bilateral oophorectomy with tumor debulk-

\footnotetext{
*Corresponding author.
}

ing. She underwent chemotherapy and external beam radiation at the time. She underwent whole pelvic radiation with 4500 cGy (centi-Gray) as well as adjuvant chemotherapy with carboplatin and paclitaxel for 6 cycles. She was then placed on hormonal therapy with Megestrol and Tamoxifen for further treatment. No metastatic disease was identified at that point. Fifteen months later, she re-presented with dysphagia, left tongue deviation, and left side weakness. MRI (magnetic resonance imaging) obtained demonstrated a large clival mass with significant erosion of the skull base extending into the jugular foramen. Based on the significant size and unusual location of tumor, we performed an endonasal biopsy to obtain a diagnosis. Tumors in this area may include metastatic disease, chordomas, chondrosarcomas, and osteosarcomas. Pathology demonstrated endometrial adenocarcinoma (Figure 1). She was then given 3500 cGy radiation dose as palliative treatment to the clival mass. Four months later, she was noted to have metastatic disease to the cervical and thoracic at that time with subsequent 3000 cGy radiation treatment to the spine (Figures 2(a)-(c)). 


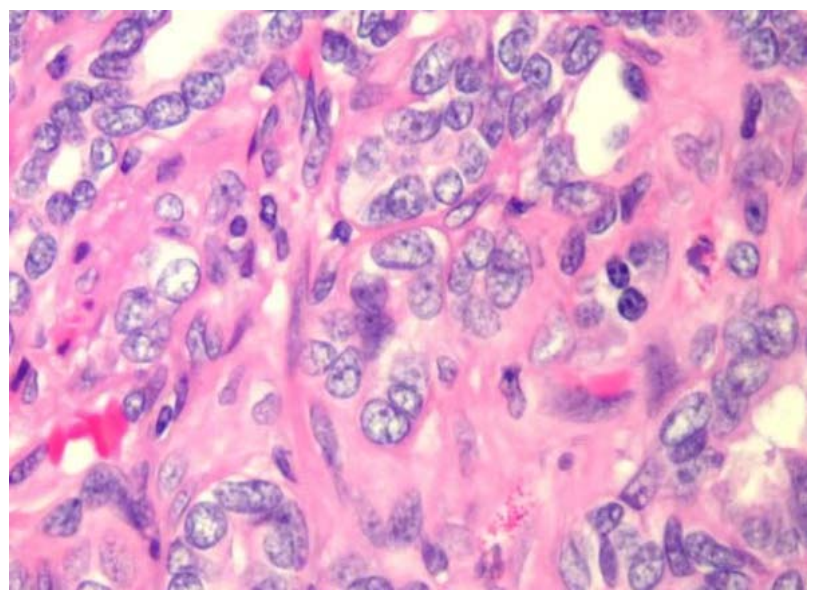

Figure 1. Hematoxyline and Eosine slide demonstrating endometrial adenocarcinoma. There are numerous, small, crowded glands with varying degrees of nuclear atypia, mitotic activity, and stratification.

\section{Results}

\section{Intraoperative}

The patient then presented once again eight months later with worsening weakness and numbness of the lower extremities, progressing to a wheelchair bound state. MRI demonstrated a pathological compression fracture at C7 with $80 \%$ loss of height and retropulsion in the spinal canal, with preservation of disc spaces (Figure 2(d)). CT imaging demonstrated near total erosion of the bone by tumor. She was taken to the operating room for a C7 corpectomy with placement of cage and anterior instrumentation. The entire C7 vertebral body was soft and eroded by tumor, and there were areas of grayish-blue tumor with some slight vascularity. The remaining vertebral body was removed in a piece-meal fashion. The patient's motor deficits and sensory deficits improved post operatively. Pathology demonstrated metastatic disease composed of numerous, small, crowded glands with varying degrees of nuclear atypia, mitotic activity, and stratification (Figure 1). Estrogen and progesterone receptor positivity was noted.

\section{Discussion}

Endometrial adenocarcinoma is the most common cancer in the female reproductive system [2]. Metastatic disease to the lungs, liver, bones and skin are quite common [2]. Methods of dissemination include local or lymphatic [3]. Bony involvement of this disease is documented to be around 10\% - 15\% [2]. Fagundes et al. did a retrospective review of 1211 patients with invasive uterine carcinoma treated with radiation alone and found that the incidence of metastasis to other organs was 56\% [4]. The incidence of metastases to the bones was $16 \%$, most

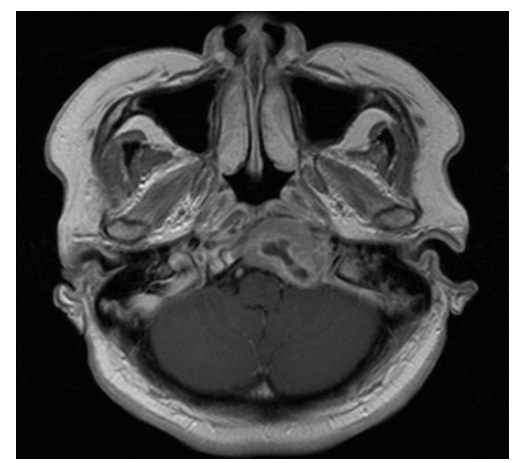

(a)

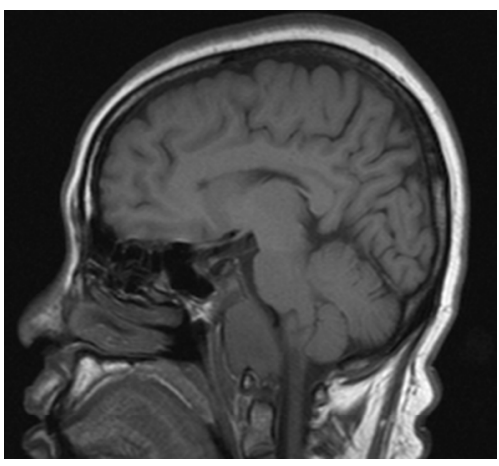

(b)

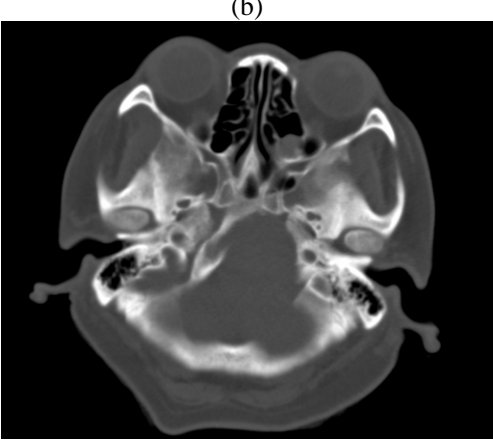

(c)

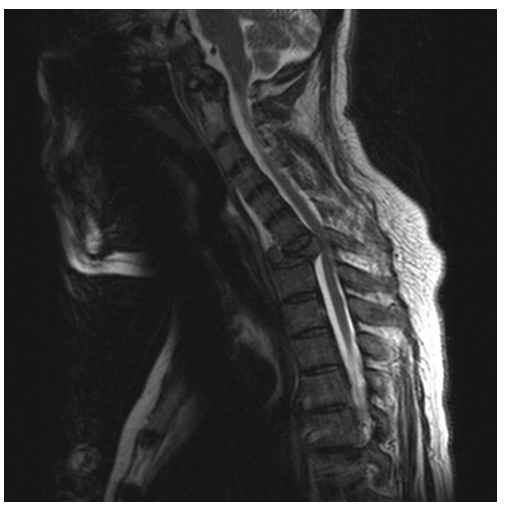

(d)

Figure 2. (a): MRI Brain demonstrating large left-sided clival mass and (b): $\mathrm{T} 1$ saggital image demonstrating clival erosion due to tumor and (c) also demonstrated on (c) CT imaging. (d): MRI c-spine demonstrating acute pathological fracture at $\mathrm{C} 7$, with severe spinal canal compression, however with preservation of disc spaces. 
commonly to the thoracic and lumbar spines [4]. There are to date only 29 reported cases of bone metastases from endometrial adenocarcinoma, although none reported in the clival skull based nor causing acute myelopathy in the cervical spine due to pathological fracture.

Albareda et al. discussed endometrial adenocarcinoma metastasis to the sacrum requiring sacrectomy with good results post operatively [2]. Al-Salam described a patient with acute onset of myelopathy due to thoracic metastasis from an endometrial stromal sarcoma with sex cord differentiation [5]. This patient underwent urgent thoracic decompression and recovered well [5]. Ye et al. studied 36 relatively healthy patients with a variety of metastatic spinal tumors to all areas of the spine who were operated on for tumor resection and spinal stabilization. They noted diminishing pain post-operatively in all patients and the following overall survival at 3 months, 6 months, 1 year, and 2 years: 97.2\%, 63.9\%, 38.9\%, $16.7 \%$ [6]. They concluded that single or multiple spinal metastases, neurological symptoms, spinal stability and patients' pre-operative condition all affect patient postoperative pain and neurological outcome [7].

Farooq et al. documented a case of a 63-year-old woman with a rapidly enlarging mass on her forehead [3]. MRI demonstrated an $8 \mathrm{~cm}$ tumor extending from the left frontal subcutaneous tissue through the skull into the epidural space, with concomitant stable spine metastases [3]. Mustafa et al. described a 45-year-old patient with scalp and left frontal bone metastases [8]. Lieschke et al. reported a case of endometrial adenocarcinoma with spread to the pituitary gland presenting as apoplexy [9]. Additionally, endometrial carcinoma has been reported to metastasize to the paranasal sinuses [10], mandible [11], and as isolated lesions in the long bones [6]. Treatment for widely metastatic endometrial adenocarcinoma is a combination of surgery, radiation, and chemotherapy with hormonal therapy as appropriate depending on receptor status of cancer [9-11]. 5 years survival for highly metastatic 4A tumors is $17 \%[9,10]$. Given this relatively favorable survival, this may argue in favor of more aggressive treatment of bone metastases in patients with advanced endometrial adenocarcinoma.

\section{Conclusion}

Endometrial adenocarcinoma must be considered on the differential diagnosis list of lesions involving the skull base and/or pathological fractures of the spine presenting acutely with spinal cord compression.

\section{REFERENCES}

[1] A. Jemal, R. Siegel, E. Ward, et al., "Cancer Statistics, 2008,” CA: A Cancer Journal for Clinicians, Vol. 58, No. 2, 2008, pp. 71-96. doi:10.3322/CA.2007.0010

[2] J. Albareda, M. Herrera, A. Salva, J. Donas and R. Gonzalez, "Sacral Metastasis in a Patient with Endometrial Cancer: Case Report and Review of the Literature," Gynecologic Oncology, Vol. 111, No. 3, 2008, pp. 583-588.

[3] M. Farooq and H. Chang, "Intracranial and Scalp Metastases of Endometrial Carcinoma," Medical Science Monitor, Vol. 14, No. 9, 2008, PP. 87-88.

[4] H. Fagundes, C. A. Perez, P. W. Grigsby, et al., "Distant Metastases after Irradiation alone in Carcinoma of the Uterine Cervix," International Journal of Radiation Oncology \& Biology \& Physics, Vol. 24, No. 2, 1992, pp. 197-204. doi:10.1016/0360-3016(92)90671-4

[5] S. Al-Salam, H. El-Terifi and S. Ghazal-Aswad, "Low Grade Endometrial Stromal Sarcoma with Sex Cord-Like Differentiation Metastatic to the Thoracic Spine," APMIS, Vol. 114, 2006, pp. 651-655. doi:10.1111/j.1600-0463.2006.apm_409.x

[6] O. Onuba, "Pathological Fracture of the Right Tibia, an Unusual Presentation of Endometrial Carcinoma: A Case Report,” Injury, Vol. 14, No. 6, 1983, pp. 541-545. doi:10.1016/0020-1383(83)90058-X

[7] S. M. Ye, X. S. Qi, Z. X. Mao, et al., "Analysis of Surgical Treatment and Effect of Spinal Metastatic Tumors," Zhongguo Gu Shang, Vol. 24, 2011, pp. 977-981.

[8] M. Mustafa, L. Al-Nuaim and N. Rahman, "Scalp and Cranial Bone Metastasis of Endometrial Carcinoma: A Case Report and Literature Review," Gynecologic Oncology, Vol. 81, No. 1, 2001, pp. 105-109. doi:10.1006/gyno.2000.6038

[9] G. J. Lieschke, B. Tress and D. Chambers, "Endometrial Adenocarcinoma Presenting as Pituitary Apoplexy,” Australian and New Zealand Journal of Medicine, Vol. 20, No. 1, 1990, pp. 81-84. doi:10.1111/j.1445-5994.1990.tb00379.x

[10] S. Ilvan, E. U. Akyildiz, Z. Calay, et al., "Endometrial Clear Cell Carcinoma Metastatic to the Paranasal Sinuses: A Case Report and Review of the Literature," Gynecologic Oncology, Vol. 94, No. 1, 2004, pp. 232-234. doi:10.1016/j.ygyno.2004.04.005

[11] D. E. Dosoretz, J. W. Orr, S. A. Salenius, et al., "Mandibular Metastasis in a Patient with Endometrial Cancer," Gynecologic Oncology, Vol. 72, No. 2, 1999, pp. 243245. doi:10.1006/gyno.1998.5223 